\section{(2) OPEN ACCESS}

\title{
Low gonorrhoea antimicrobial resistance and culture positivity rates in general practice: a pilot study
}

\author{
Maartje Visser (ㄷ) ' Mireille van Westreenen, 2,3 Jan van Bergen, 1,4,5 \\ Birgit $H$ B van Benthem ${ }^{1}$
}

- Additional material is published online only. To view please visit the journal online (http://dx.doi.org/10.1136/ sextrans-2019-054006).

${ }^{1}$ Centre for Infectious Disease Control, National Institute for Public Health and the Environment (RIVM), Bilthoven, The Netherlands

${ }^{2}$ Department of Medical Microbiology and Infectious Diseases, Erasmus MC, Rotterdam, The Netherlands ${ }^{3}$ STAR-SHL Medical Diagnostic Centre, Rotterdam, The Netherlands

${ }^{4}$ STI AIDS Netherlands, Amsterdam, The Netherlands ${ }^{5}$ Division of Clinical Methods and Public Health, Department of General Practice, Amsterdam UMC Location AMC Amsterdam, The Netherlands

\section{Correspondence to} Maartje Visser, Centre for Infectious Disease Control, National Institute for Public Health and the Environment (RIVM), Bilthoven 3720 BA, The Netherlands; maartje.visser@ rivm.nl

Received 11 February 2019 Revised 10 April 2019 Accepted 16 April 2019 Published Online First 30 April 2019

\section{Check for updates}

(c) Author(s) (or their employer(s)) 2020. Re-use permitted under CC BY-NC. No commercial re-use. See rights and permissions. Published by BMJ.

To cite: Visser $M$, van Westreenen $M$, van Bergen J, et al. Sex Transm Infect 2020:96:220-222

\begin{abstract}
Objective In the Netherlands, the Gonococcal Resistance to Antimicrobials Surveillance (GRAS) programme is carried out at Centres for Sexual Health (CSH), which provide care for sexual high-risk populations. However, half of gonorrhoea infections are diagnosed in general practice (GP). We performed a pilot study to explore expanding GRAS to GPs using laboratory-based surveillance. Additionally, antimicrobial resistance patterns of GP and CSH patients were compared.
\end{abstract}

Methods Three laboratories from different regions were included, which all perform gonorrhoea diagnostics for GPs and used ESwab for patient sampling. Additional culturing for all GP patients with gonorrhoea took place from February to July 2018. After positive PCR-nucleic acid amplification test, residual ESwab material was used for culture. In positive cultures, susceptibility testing was performed for azithromycin, ciprofloxacin, cefotaxime and ceftriaxone using Etest.

Results During the study period, 484 samples were put in culture. $16.5 \%$ of cultures were positive $(n=80)$. Antimicrobial resistance levels were low, with $2.6 \%$ resistance to azithromycin, $21.5 \%$ to ciprofloxacin and $0.0 \%$ to cefotaxime and ceftriaxone. Resistance levels in CSH GRAS data (first half of 2018) were 19.2\% for azithromycin, 31.5\% for ciprofloxacin, 1.9\% for cefotaxime and $0.0 \%$ for ceftriaxone.

Conclusions Culture positivity rates for GP patients were low, probably due to long transportation times and awaiting PCR test results before attempting culture. Positivity rates might be improved by making changes in sampling and/or transportation methods, but that would require involvement of GPs and patients instead of keeping the surveillance lab based. Resistance levels appeared to be lower at GPs than at the $\mathrm{CSH}$, indicating that resistance might emerge first in more high-risk populations. It is important to consider all potentially relevant patient populations when establishing a gonococcal antimicrobial resistance surveillance programme. However, based on the findings from this study the current GRAS programme will not be extended to GPs.

\section{INTRODUCTION}

Neisseria gonorrhoeae infection (gonorrhoea) is one of the most common STIs in the Netherlands. In the past, N. gonorrhoeae has shown to be extremely capable of developing antimicrobial resistance, threatening the availability of effective treatment. ${ }^{1}$ Resistance to the current first-line antibiotic ceftriaxone has not yet been reported in the
Netherlands, ${ }^{2}$ but has been seen in other countries, including the UK. ${ }^{3}$

To monitor gonorrhoea antimicrobial resistance in the Netherlands, the Gonococcal Resistance to Antimicrobials Surveillance (GRAS) programme was established in 2006. GRAS is a sentinel surveillance system including 18 out of 24 Centres for Sexual Health (CSH). However, more than half of gonorrhoea diagnoses in the Netherlands are carried out in general practice (GP) (in 2016: $6092 \mathrm{CSH}$ diagnoses vs approximately 9000 GP diagnoses). ${ }^{2}$ Thus, many patients are not included in GRAS. Currently, it is unknown to what extent antimicrobial resistance patterns among CSH visitors are representative for patients who visit the GP for STI testing. Gaining insight in the gonococcal antimicrobial resistance among GP patients could therefore provide useful information and will allow for a more comprehensive understanding of the current antimicrobial resistance patterns of gonorrhoea in the Netherlands.

In GP, gonorrhoea diagnoses are based on PCRnucleic acid amplification test (NAAT). STI testing guidelines for GPs are similar to those at the CSH, but it is known that, for example, extragenital testing is less often performed by GPs. ${ }^{4}$ Culture is also not routinely performed, but is necessary to determine antimicrobial susceptibility. Because it requires more effort from GPs and their patients to collect additional samples for culturing, we first wanted to explore implementation of a laboratorybased surveillance that requires no additional sample collection. Therefore, the primary goal of this pilot study was to explore the feasibility of a laboratory-based GP surveillance of gonococcal antimicrobial resistance. Additionally, we aim to describe antimicrobial resistance patterns of patients with gonorrhoea in GP, and compare these to patterns of CSH patients.

\section{METHODS}

Laboratories were eligible for inclusion in the pilot study if they performed gonorrhoea diagnostics for GPs, if they were equipped to perform culture and susceptibility testing for N. gonorrhoeae and if they used ESwab for collection and transportation of samples. ESwab is a collection and transport system that contains enough liquid sample suspension to perform multiple tests on one specimen. It is therefore possible to first perform PCR-NAAT diagnostics, and if positive for gonorrhoea perform culture 


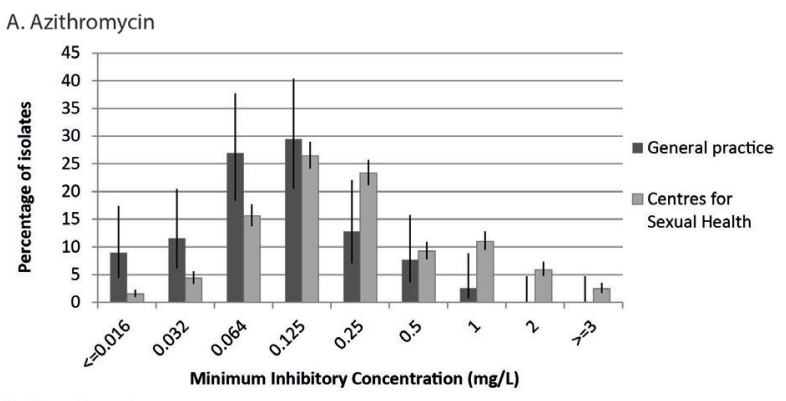

B. Ciprofloxacin

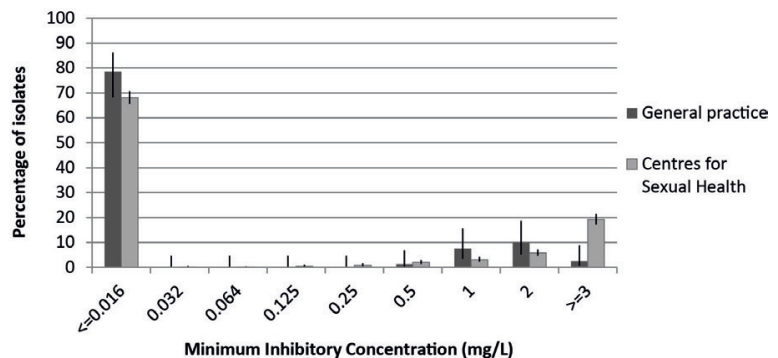

C. Cefotaxime

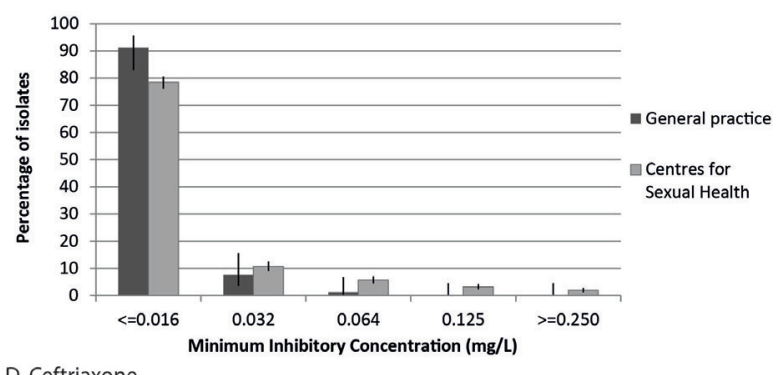

D. Ceftriaxone

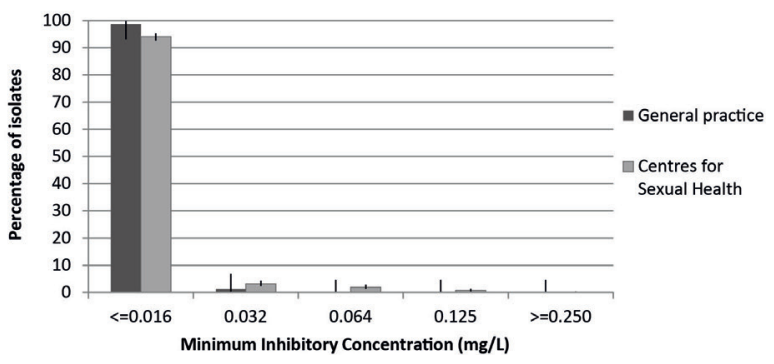

Figure 1 Minimum inhibitory concentration (MIC) value distributions for (A) azithromycin, (B) ciprofloxacin, (C) cefotaxime and (D) ceftriaxone susceptibility of Neisseria gonorrhoeae from the Gonococcal Resistance to Antimicrobials Surveillance (GRAS) in general practice pilot study and from the GRAS programme at Dutch Centres for Sexual Health in the first half of 2018.

using the same material. Three large laboratories participated in this study: one from an urban region (STAR-SHL) in the southwest of the Netherlands, and two from rural regions in the north of the Netherlands (Certe and Izore).

The three laboratories collected data from GP patients that tested positive for gonorrhoea from February to July 2018. After positive NAAT, ESwab material was used for culture. In case of a positive culture, antimicrobial susceptibility testing was performed using Etest for four antibiotics: azithromycin, ceftriaxone, ciprofloxacin and cefotaxime. The laboratories Certe and Izore did not culture urine samples and samples with a cycle threshold value over 30 due to low chance of succeeding. All labs reported data from both culture positive and negative patients, including sex, age, anatomical location of sampling, result of culture and results from antimicrobial susceptibility testing (minimum inhibitory concentration (MIC) values).

Materials used for sample collection and culture differed between the laboratories. Swabs used were ESwab from Copan (Certe) and BD (Izore), and the $\Sigma$-Transwab (STAR-SHL), all containing liquid Amies medium. Plates used for culture were for Certe chocolate agar and chocolate agar+VCTA (Mediaproducts), for STAR-SHL Neisseria selective medium PLUS (Thermo Scientific), and for Izore Chocolate Agar (GC II Agar with IsoVitaleX) (BD), Neisseria Selective Medium PLUS (Thermo Scientific) and Mueller Hinton Chocolate Agar (BD).

Descriptive analyses were used to calculate culture positivity rates and the percentage of antimicrobial resistance. Antimicrobial resistance was defined using MIC breakpoints from the European Committee on Antimicrobial Susceptibility Testing (azithromycin $>0.5 \mathrm{mg} / \mathrm{L}$, ciprofloxacin $>0.06 \mathrm{mg} / \mathrm{L}$ and cefotaxime and ceftriaxone $>0.125 \mathrm{mg} / \mathrm{L}) .^{5}$ Univariate logistic regression analyses were used to assess differences in culture positivity rates by patient characteristics. Age was included in three categories $(<25,25-34,>34)$. Multivariate analyses were not possible due to low numbers. We compared the antimicrobial resistance patterns from GP patients with data from the regular GRAS surveillance at CSH from the first half of 2018 using Fisher's exact test.

\section{RESULTS}

A total of 469 GP patients tested positive for gonorrhoea at the three laboratories between February and July 2018: 323 at STAR-SHL, 77 at Certe and 69 at Izore. Some patients had samples from multiple anatomical locations. Therefore, culture was performed on 484 samples, of which 80 (16.5\%) were positive. All analyses were performed on sample level. Most samples were urogenital $(n=395)$, followed by anal $(n=42)$ and oral $(n=29)$. Eighteen samples were of unknown anatomical location. Of the three laboratories, Izore had a significant lower culture positivity rate $(8.2 \%$ positive; OR $0.40,95 \%$ CI 0.17 to 0.98$)$ compared with STAR-SHL (18.1\%) and Certe (17.5\%). The anatomical location of the sample was also significantly associated with culture positivity. Compared with vaginal swabs (16.2\% culture positive), anal swabs were less likely to result in a positive culture $(2.4 \%$ positive; OR $0.13,95 \%$ CI 0.02 to 0.95$)$, as were oral swabs $(0.0 \%$ positive). Cultures from urine samples showed a significantly higher chance of succeeding $(25.7 \%$ positive; OR $1.80,95 \% \mathrm{CI}$ 1.02 to 3.18 ). Age and sex were not significantly associated with culture positivity in univariate analyses. See also online supplementary table 1 . The $16.5 \%$ culture positivity rate at the GPs was lower than the average $54.7 \%$ culture positivity rate at the $\mathrm{CSH}$ in the first half of 2018 .

Antimicrobial susceptibility testing was performed for the 80 positive cultures. Resistance levels were low, with $2.6 \%$ resistant to azithromycin, $21.5 \%$ to ciprofloxacin and $0.0 \%$ to cefotaxime and ceftriaxone. Resistance levels in CSH GRAS data (first half of 2018 ) were $19.2 \%$ for azithromycin, $31.5 \%$ for ciprofloxacin, $1.9 \%$ for cefotaxime and $0.0 \%$ for ceftriaxone. This difference was statistically significant for azithromycin $(\mathrm{p}<0.001)$, but not for ciprofloxacin $(p=0.078)$ and cefotaxime $(p=0.392)$. The MIC distributions including 95\% CI from the GP population and the CSH population are shown in figure 1.

\section{DISCUSSION}

This study piloted a laboratory-based antimicrobial resistance surveillance for GP patients, without GPs or patients being involved. Culture positivity rates were low, and antimicrobial 
resistance rates were lower than the rates seen in the GRAS programme at $\mathrm{CSH}$.

The main limitation of this study was the small sample size. As this was a pilot study, we only enrolled the surveillance in three regions. We did however include both urban and rural laboratories, so the findings are expected to be representative for other regions in the Netherlands as well. Due to the low culture positivity rates, the amount of samples available for susceptibility testing was limited. Therefore, extensive statistical analyses could not be performed. Furthermore, due to the low culture positivity rate, the representativeness of the results of the susceptibility testing should be interpreted with caution.

The low GP culture positivity rate could be explained by the fact that in the pilot study PCR testing results had to be available before samples were put in culture, and as bacterial viability in a transport medium decreases over time, ${ }^{6}$ this delay might have caused decreases in culture positivity rates. According to the CSH GRAS protocol, PCR test results do not have to be awaited, and samples are put immediately in culture when received at the laboratory. In addition, transportation times from GP to laboratory could have been longer than at the CSH because GP practices are often further away from the laboratory, and because GPs did not know the samples would be used for culturing and therefore put in no additional efforts to ensure the samples were sent to the laboratory as soon as possible. The time between sample collection at GP and culturing was unknown at the laboratories. Samples were in all three laboratories collected from the GPs once per day. Estimations of time between sample collection and sample testing varied from a few hours to more than a day.

Results from this study also showed that the anatomical location of the sample was strongly associated with culture positivity. Anal and oral samples had a lower chance of resulting in positive culture, which has been seen before. ${ }^{7-9}$ The finding that urine samples had a higher chance of succeeding was unexpected, since two laboratories said not to culture urine samples due to low expected culture positivity. Despite the fact that this finding is only based on univariate analysis, it does indicate that urine could also be used for culturing.

Antimicrobial resistance rates were compared between the GP and $\mathrm{CSH}$ populations. For all antimicrobials, resistance levels were lower among GP patients. These findings were only statistically significant for azithromycin, which could be due to the small sample size, but it gives an indication that antimicrobial resistance might occur more often among CSH visitors. This could be explained by the fact that 'core groups' with high risk of STI are known contributors to dissemination of antimicrobial resistant gonorrhoea, ${ }^{10}$ and $\mathrm{CSH}$ provide care especially for groups at high risk for STI, while the GP population is generally at lower risk.

The main aim of this study was to explore the feasibility of a laboratory-based GP surveillance of gonococcal antimicrobial resistance. It was possible to perform surveillance without engaging GPs and/or their patients, but it resulted in low culture positivity rates. For a more comprehensive and complete surveillance system the culture positivity rates should be improved, which is likely only possible with the involvement of GPs and patients. It is, however, undesirable to burden GPs with antimicrobial surveillance, as GPs are already under time pressure, and are not expected to perform public health surveillance tasks. Furthermore, the low antimicrobial resistance rates among GP patients indicate that antimicrobial resistance is more prevalent among CSH visitors, which are already well covered by the existing national GRAS surveillance. This also makes the establishment of a GP antimicrobial surveillance less urgent.
In conclusion, it is important to consider all potentially relevant patient populations when establishing a gonococcal antimicrobial resistance surveillance programme. However, the current findings indicate that additional efforts are needed to establish antimicrobial surveillance in GP practice, while resistance rates are low. Based on the findings from this study the current GRAS programme in the Netherlands will therefore not be extended to the GP population.

\section{Handling editor Claudia S Estcourt}

Acknowledgements The authors thank the Laboratories STAR-SHL, Izore and Certe and their staff for the collaboration and participation in the pilot study, and the GRAS board for their contributions to the study design and interpretation of the results.

Contributors All authors were involved in establishing the study design. MV coordinated the execution of the study, analysed the data and drafted the manuscript. All authors contributed to interpretation of the results and commented on the manuscript. All authors read and approved the final manuscript.

Funding This study was funded by the Dutch Ministry of Health, Welfare and Sport. Competing interests None declared.

\section{Patient consent for publication Not required.}

Ethics approval This study used anonymised data collection without the involvement of the patient. This study was declared to fall outside the scope of the Dutch law on medical scientific research with human subjects (WMO) by the Medical Ethical committee of the Erasmus Medical Centre in Rotterdam (MEC-2017-570). Therefore, further ethical approval was not required.

Provenance and peer review Not commissioned; externally peer reviewed.

Data availability statement Data from this study are not publicly available, but may be provided for scientific purposes upon reasonable request.

Open access This is an open access article distributed in accordance with the Creative Commons Attribution Non Commercial (CC BY-NC 4.0) license, which permits others to distribute, remix, adapt, build upon this work non-commercially, and license their derivative works on different terms, provided the original work is properly cited, appropriate credit is given, any changes made indicated, and the use is non-commercial. See: http://creativecommons.org/licenses/by-nc/4.0/.

\section{ORCID iD}

Maartje Visser http://orcid.org/0000-0002-2607-7677

\section{REFERENCES}

1 Unemo M, Del Rio C, Shafer WM. Antimicrobial resistance expressed by Neisseria gonorrhoeae: a major global public health problem in the 21st century. Microbiol Spectr 2016;4

2 Visser M, Van Aar F, Op de Coul E. Sexually transmitted infections in the Netherlands in 2017 RIVM; 2018.

3 Public Health England. Health protection report vol.12 no.11. UK case of Neisseria gonorrhoea with high-level resistance to azithromycin and resistance to ceftriaxone acquired abroad Public Health England; 2018.

4 den Heijer CDJ, Hoebe CJPA, van Liere GAFS, et al. A comprehensive overview of urogenital, anorectal and oropharyngeal Neisseria gonorrhoeae testing and diagnoses among different STI care providers: a cross-sectional study. BMC Infect Dis 2017;17.

5 European Committee on Antimicrobial Susceptibility Testing. Breakpoint tables for interpretation of MICs and zone diameters. version 8.1, 2018. Available: http://eucast. org/clinical_breakpoints/ [Accessed Jan 2019].

6 Papp JR, Henning T, Khubbar M, et al. Recovery of Neisseria gonorrhoeae from 4 commercially available transport systems. Diagn Microbiol Infect Dis 2016;86:144-7.

7 Cornelisse VJ, Chow EPF, Huffam S, et al. Increased detection of pharyngeal and rectal gonorrhea in men who have sex with men after transition from culture to nucleic acid amplification testing. Sex Transm Dis 2017;44:114-7.

8 Mohammed $\mathrm{H}$, Ison CA, Obi C, et al. Frequency and correlates of culture-positive infection with Neisseria gonorrhoeae in England: a review of sentinel surveillance data. Sex Transm Infect 2015;91:287-93.

9 Wind CM, de Vries HJC, Schim van der Loeff MF, et al. Successful combination of nucleic acid amplification test diagnostics and targeted deferred Neisseria gonorrhoeae culture. J Clin Microbiol 2015;53:1884-90.

10 Lewis DA. The role of core groups in the emergence and dissemination of antimicrobialresistant $N$ gonorrhoeae. Sex Transm Infect 2013;89 Suppl 4:iv47-51. 